\title{
Barriers to the Adoption and Use of Personal Health Record Systems
}

\author{
Leslie S. Liu, Patrick C. Shih, Gillian R. Hayes \\ Department of Informatics \\ Donald Bren School of Information and Computer Sciences \\ University of California, Irvine \\ Irvine, CA 92697-3440, USA \\ \{lsliu, patshih, gillianrh\}@ics.uci.edu
}

\begin{abstract}
Personal health records (PHR) have enormous potential to improve both documentation of health information and patient care. The adoption of these systems, however, has been relatively slow. In this work, we used a multi-method approach to evaluate PHR systems. We interviewed potential end users - clinicians and patients - and conducted evaluations with patients and caregivers as well as a heuristic evaluation with HCI experts. In these studies, we focused on three PHR systems: Google Health, Microsoft HealthVault, and WorldMedCard. Our results demonstrate that both usability concerns and socio-cultural influences are barriers to PHR adoption and use. In this paper, we present those results as well as reflect on how both PHR designers and developers might address these issues now and throughout the design cycle.
\end{abstract}

\section{Categories and Subject Descriptors}

J.3 Computer Applications: Life and Medical Sciences; H.4 Information Systems Applications; H.1 Models and Principles.

\section{General Terms}

Human Factors.

\section{Keywords}

Personal health records, usability evaluation.

\section{INTRODUCTION}

In the United States and in many other countries, it can be tedious for patients to obtain copies of their official health records from service providers, making it difficult for patients to seek second opinions, change providers, or even just feel in control of their own information. Furthermore, records that patients create themselves tend not to be included in the official patient record.

Permission to make digital or hard copies of all or part of this work for personal or classroom use is granted without fee provided that copies are not made or distributed for profit or commercial advantage and that copies bear this notice and the full citation on the first page. To copy otherwise, to republish, to post on servers or to redistribute to lists, requires prior specific permission and/or a fee.

iConference 2011, February 8-11, 2011, Seattle, WA, USA.

Copyright (C) 2011 ACM 978-1-4503-0121-3/11/02 ...\$10.00
Thus, a patient's view of his or her records-and potentially health status - may be substantially different from that of the providers. Having a personal health records (PHR) system that is maintained by patients and their families and shared with clinicians can support the maintenance of accurate and complete health records.

These PHR records are often created and monitored by the patients themselves. They can be desktop-based, Internet-based, or mobile (e.g., located entirely on a mobile phone or portable storage device). Krohn suggests that there are four different types of PHR models: stand-alone, health plan patient portals, Electronic Medical Record (EMR) patient portals, and consumercentric PHR [9]. If properly maintained and up-to-date, these systems can help a patient communicate important health information with clinicians. In the United States, however, only 7 million adult patients make use of PHR [13]. The enormous potential of these systems when coupled with their limited adoption have left many researchers, clinicians, and public health officials pondering the disconnect [2].

Specifically, this research sets out to understand the particular challenges limiting the adoption and use of PHR through a systematic evaluation of three Internet-based, consumer-centric PHR systems. These systems were chosen from various types because they represented a wide variety of features and functionality as well as being easily accesible to those with Internet and are a fast growing sector of Health IT in recent years. Furthermore, many individuals argue that consumer-centric Health IT is key to the future of quality improvement in healthcare moving forward (e.g., http://www.projecthealthdesign.org). We first evaluated the three PHR systems by asking end-users-patients and their caregivers - to accomplish a list of tasks that mimic real world usage using these systems. We then interviewed these end-users as well as clinicians who were all potential users of these systems but had not adopted them. A variety of issues such as advanced computational functionality of PHR, terminology, personalization, familirty and comfort, collaboration, privacy and security, and ensuring accurate data were also brought by both clinicians and end-users. In order to complement these two methods (end-user evaluation and interviews) and recognizing that many hospitals and clinics lack the resources to conduct substantial usability evaluations in practice, we focused on the use of a discount usability method, heuristic evaluation [4], for PHR. 
In this work, we provide analysis of empirical data drawn from multiple evaluation approaches. This analayis focuses on the usability and usefulness of PHR, which begins to explain the limited adoption of these systems. The remainder of this paper is structured as follows. First, we lay out related work on healthcare usability and evaluation. Next, we describe the methods we used in gathering and analyzing our empirical data and present the results of these studies. We close with a discussion of the ways in which different methods enabled us to uncover different issues, further supporting the need for multi-method approaches to designing and evaluating Health IT. Finally, we close with an examination of future directions for reseearch.

\section{RELATED WORK}

Past research on PHR has typically focused on the definition and features of PHR systems and the potential benefits and limitations of using such systems from a health outcomes perspective, such as supporting patient-provider communication [17]. In these studies where researchers required patients to use these systems as part of the study, such medical benefits were that collaborative disease tracking was able to lower communication barriers between patients and their caregivers. Similarly, patients who were more engaged in their health were often more active in working with their providers in thereupitc alliance [17].

However, there is little work that addresses what happens when patients and clinicians are not required to use PHR by a research team. The work that has been done has focused mainly on the usability and utility of PHR from a patient's point of view, but less centered on the socio-cultural aspects of PHR [14]. Those studies that have been published have mainly focused on surveying potential users about PHR [5, [20]. For example, according to one survey, $50 \%$ of the people older than 65 reported that they would prefer a paper PHR over an online version, while only $20 \%$ of those under 65 said they would prefer a paper PHR [20], indicating that perhaps as computer savvy younger individuals age and require more healthcare, PHR adoption may increase. Some studies have also proactively focused on the potential challenges and appropriate models for PHR, such as four different PHR models (stand-alone, health plan, EMR, and consumer-centric) $[9,16]$. Similarly in a study done by Win et al., they suggest that PHR developers need to more deeply consider potential problems such as handling and designing for security, confidentiality, and availability [19]. To date, there have been limited evaluations conducted of specific applications of PHR technology $[3,8,10]$. Although these are useful for understanding some of the issues associated with PHR, they largely focus on a specific perspective (e.g. nurses, patients) and are most concerned with health outcomes or specific technological issues. For example, in one study, Lee et al. conducted an evaluation of a novel PHR system that was developed by the authors from a nursing perspective [10]. They found that the actual content that was in the PHR could be very useful for healthcare providers. Furthermore, PHR systems could be useful as educational guidelines for nurse practitioners [10]. In this work, we build on this related literature by focusing on issues of PHR when considering use by multiple stakeholders.

Across the existing body of research, some indicators about the general challenges of PHR adoption begin to emerge. Limitations of the technology itself-including usability concerns-may hinder both patients and clinicians from fully reaching the goals they set out for these systems. Limited standards exist for the implementation and use of health information technologies and record-keeping systems, creating a systemic problem of interoperability. Likewise, the substantial regulatory process and concerns about privacy and security (e.g., HIPAA ${ }^{1}$ ) may enact further barriers as institutions try to deploy these systems within their organizational contexts. Finally, PHR add an additional challenge to those records systems focused on the use in clinics: they put patients and caregivers "in charge" of the creation and maintenance of electronic data that then may not be medically relevant nor acceptable to professionals.

\section{METHODS FOR UNDERSTANDING PHR ADOPTION AND ACCEPTANCE}

To understand the challenges to adoption and use of PHR, we evaluated three specific consumer-centric PHR systems: Google Health, Microsoft HealthVault, and WorldMedCard, now known as WorldHealthRecord. These were chosen from a field of dozens based on the following criteria:

- Available to the public, not just patients of a particular healthcare system (e.g, Blue Cross or Veterans Affairs)

- Internet-based

- Mix of familiar and less known corporations

In this work, we took a multi-method approach to understanding the barriers to adoption and use of PHR. We conducted end-user evaluations and clinician interviews in order to understand some of the issues that arise in the lack of PHR adoption. We further conducted a heuristic evaluation of these sites to examine the usability concerns experts might raise.

\subsection{End-User Evaluations}

We conducted a counter-balanced within subjects evaluation of the three PHR systems with 18 end-users with a wide range of technical and medical experience. The participants were aged from 18 to 55 years old and hold a variety of careers, from medicine to business to engineering. End-users were presented with a list of 9 specific tasks ( 3 tasks per website), all to be performed on each site using standardized reference patient data. These tasks were designed to mimic the types of everyday activities in which end-users might engage. They were asked in various scenarios to consider themselves as either patients or caregivers (e.g., act as a parent storing data for the entire family). To evaluate the full range of activities typically employed by patients, these tasks assumed patients are not necessarily using a PHR that is connected directly to an EMR updated by their providers. For example, they were asked to find and print copies of particular records to bring to a physician. They were also asked to update information and upload images (such as an X-Ray they might have been handed at a physical meeting with a physician). On-screen actions were recorded with screen capture software during each task. Observing and capturing the end-users' onscreen actions allowed us to thoroughly understand hidden technical usability issues that may not have been evident from the interviews. It also allowed us to understand basic technical issues that were present to a range of end-users from those who were computer-savvy to those who were not. Similarly, observations of use were able to present basic medical issues that were obvious to

\footnotetext{
${ }^{1}$ http://www.hhs.gov/ocr/privacy/, Retrieved March 2009
} 
end-users familiar in medicine and to those who were not. On average, it took approximately one hour for an end-user to complete the task list.

\subsection{Interviews}

Following the end-user evaluation sessions, we conducted interviews with each of the 18 participants. In these interviews, we focused on how these potential end-users might take up PHR in their own lives, as patients, caregivers, or both. Participants also expressed their general thoughts on PHR, including their beliefs about privacy, security, computing systems, and so on.

We also conducted semi-structured interviews with five clinicians about the three PHR systems used in the study and their views of PHR generally. The clinicians included an emergency medicine physician, a pediatrician, a registered nurse, an internal medicine physician, and a family practice physician. All clinicians were given a list of the end-user tasks for reference and access to all PHR services prior to the interview. Topics that were discussed throughout the interview were whether the PHR systems presented in this study would be useful in a clinician-to-patient scenario and the clinicians' general viewpoints on electronic health data and PHR systems. Clinicians also described their views of what it would be like if patients were to give them information from PHR services. The clinician interviews allowed us to examine socio-cultural and medical issues, such as privacy and trust or clinical relevancy. The interviews also gave insight on how PHR might be usefully employed by clinicians. The interviews lasted between twenty minutes and an hour, and sometimes included follow-up by other means (e.g., email) when clinical demands required cutting the interview short. All the interviews were audio-recorded and transcribed for analysis.

\subsection{Heuristic Evaluation}

We conducted a heuristic evaluation of the three PHR systems with five HCI experts (graduate students and professors who specialize in HCI). Each expert had the end-user task list for reference. The expert logged into each of the PHR systems to evaluate the specific system based on Nielsen's set of heuristics ${ }^{2}$. For each heuristic, experts documented on paper how they believed the heuristic and the actual system matched up. On average, this process for all three PHR systems took a total of approximately an hour to an hour and a half. At the end of each evaluation session, the HCI experts were encouraged to give general feedback regarding their perceptions of PHR systems. The heuristic evaluation session was more effective in uncovering usability bugs of technical nature than the end-user evaluations and interviews and allowed us to explore a breadth of issues on PHR use and adoption together with the aforementioned methods.

\subsection{Analysis}

For both the end-user and the clinician groups, we documented and grouped issues from the evaluations to uncover relevant themes. We then examined the results of each interview group collectively to understand themes that were present in both enduser evaluations and clinician interviews. We documented the usability bugs that arose in the end-user evaluation and compared those to the bugs identified by experts during the heuristic evaluation. We then revisited the interview data to understand, in

\footnotetext{
2 http://www.useit.com/papers/heuristic/heuristic_list.html, Retrieved March 2009
}

more depth, those issues that arose both in the evaluation study and in the examination of the heuristic evaluation session.

\section{CHALLENGES AND BARRIERS}

Our results indicate that substantial usability challenges and the complexity of PHR systems can lead end-users to believe they "may not actually save time" [EU14 $\left.{ }^{3}\right]$ or that they would only be useful if a patient has a "serious health condition" [EU16]. Furthermore, both patients and clinicians expressed apprehension about the potential for patient-created mistakes in the medical data resulting from the complexity of these systems. This apprehension can lead some people to support the idea of "read-only" PHR, which patients and their caregivers could use for "doublechecking" of medical data [EU4]. The needs of clinicians and patients may inherently be at odds in these systems that are built for both groups. Many end-users reported that PHR were too complicated. However, on the other hand, clinicians described concerns about the over-simplified view presented in these systems. Even those physicians in favor of patient-managed data were worried about the balance of burden and benefit. For example:

“...most of that information is really pretty easy to get on a first visit. Especially if they're...conscious. ...the only advantage would be if a patient kept it up to date... But [the PHR systems shown during the study] almost seemed too simplistic..." [MP1]

A variety of challenges have hindered PHR adoption and acceptance. To realize the full potential of PHR systems, we must first understand and address these hurdles. In this section, we describe the themes that emerged in our evaluations that indicate challenges to PHR use.

\subsection{Advanced Computational Functionality}

Balancing an appropriate amount of functionality with simplicity of use can be a challenge for any interactive system. However, given the complexity of medical records and the systems used to represent them, this challenge becomes even more acute for PHR and other Health IT. The three systems evaluated in this work all had similar core functionality of maintaining patient health data but each had a variety of features unique only to them. For example, Microsoft HealthVault provided users with the ability to electronically "share", which allowed them to give access to their PHR to certain people. Google Health offered a function to search for a local physician within a specific medical field. WorldMedCard allowed users to input insurance information as part of their PHR.

Despite the large feature lists across all three systems, both clinicians and end-users often suggested additional features during interviews. For example, a primary care physician requested graphing and medical decision support:

"The [designers of PHR] that are creating these need to
figure out...[how] to interpret the data
electronically...say a potassium level... means the same
thing to everybody, and so once you have all of this data,
and you can actually graph it over time." [MP1]

\footnotetext{
${ }^{3}$ EU denotes end-users, typically patients and their caregivers; MP denotes clinicians; HE denotes HCI experts.
} 
On the other hand, extensive functionality and information were also recognized as potential problems. PHR, largely meant to be comprehensive health records systems, can be too comprehensive to users that only use a subset of features.

"The layout is so cluttered; things are not separated by boxes or anything, I'm not even sure if information is separate from one another or not." [EU10]

For example, the inclusion of billing or insurance information, patient-entered dietary and exercise information, and physicianentered clinical data on the same page was confusing to many end-users.

The end-users recognized categorization and collapsing menus as helpful in reducing confusion and finding needed content. The exact contents of those categories, however, is an open challenge; participants' opinions varied in how medically-aligned they wanted these categories. For example, one end-user commented that at the time of the evaluation, "I don't like how Google Health only has one set of diseases" [EU8].

\subsection{Terminology}

Many end-users noted that specific information could be confusing for users without medical training (e.g., specific descriptions of diseases). The "medical jargon" [EU8] and advanced language were often noted to be too complex and more likely a "match with hospital records than with what a patient would think" [HE5]. These results are consistent with other research that demonstrates the challenges of low health literacy on patients' ability to interpret medical information. For example, in one study, many patients (regardless of their native tongue) could not even read the instructions on their medication bottles, let alone explain how to take their medication correctly [18]. Thus, our results suggest that specific medical jargon, particularly in applications that are geared toward the general population like PHR - who typically are not fluent in medical terminologyshould be kept as basic as possible. For example, in the case of diabetes, most of the general public knows about diabetes and can distinguish between Type 1 and Type 2 diabetes, but the PHR sites we included in our evaluation were much more specific in their categorization. Google Health, for example, at the time of evaluation, listed extensive diabetes options: diabetes insipidus; diabetes insipidus, nephrogenic; diabetes mellitus, type 2; central diabetes insipidus; gestational diabetes; and diabetes, type 1. These sorts of categories can confuse someone without medical training and could potentially be harmful if the patient enters data incorrectly or selects the less appropriate choice - a concern for both patients and clinicians in our interviews.

Given the complexity of medical information, recognition of medical terms over recalling them also emerged as a primary, though somewhat unsurprising, concern in our evaluations. The ability to search within different listings for various diseases, hospitals, and clinicians were nearly universally noted as being important to successful use of PHR by patients. For patients, the ability to search for specific diseases, hospitals, and clinicians based on certain descriptions was reported to be much more preferable than requiring users to type in this information directly as some medical records systems do.

\subsection{Personalization}

The aesthetics of the different PHR services in our study varied greatly. Google, known for its clean and simplistic look, replicated the simplistic style in Google Health. As featured in other Google applications, the sidebar of categories seen in most other Google applications was also present in Google Health (see Figure 1). Microsoft HealthVault also utilized the branding and color scheme typically found in other Microsoft products. Users reported that HealthVault had a "Windows feel" to it, with much more text than the simplistic design that Google chooses to employ (see Figure 2). In WorldMedCard, large icons near the top of the screen symbolized different categories. The overall WorldMedCard, look and feel included bright colors not represented in the other systems (see Figure 3).

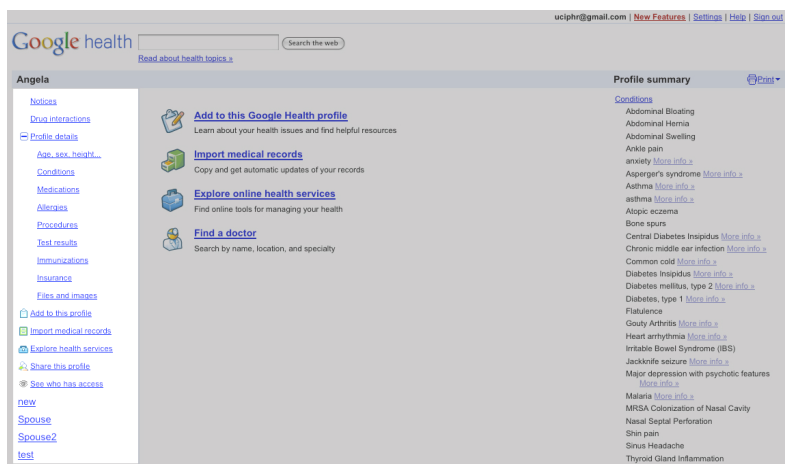

Figure 1: The sidebar (highlighted, left) of categories in Google Health is consistent with the Google look

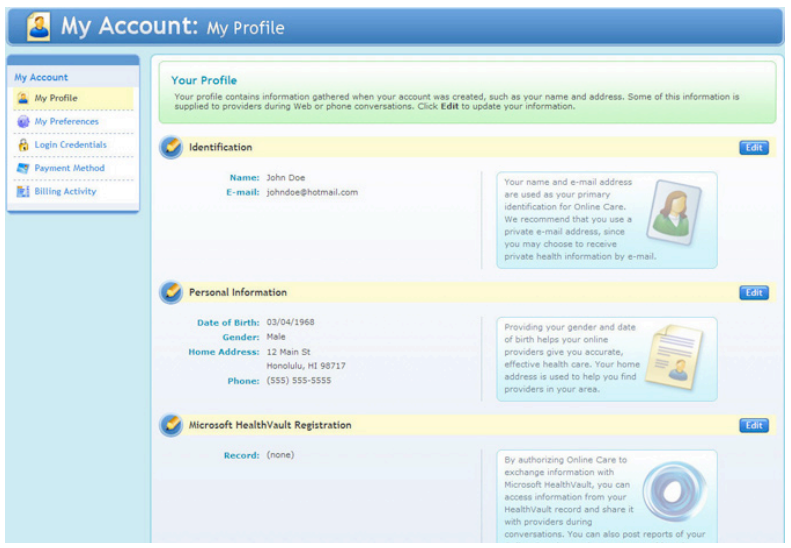

Figure 2: HealthVault includes more text than other systems.

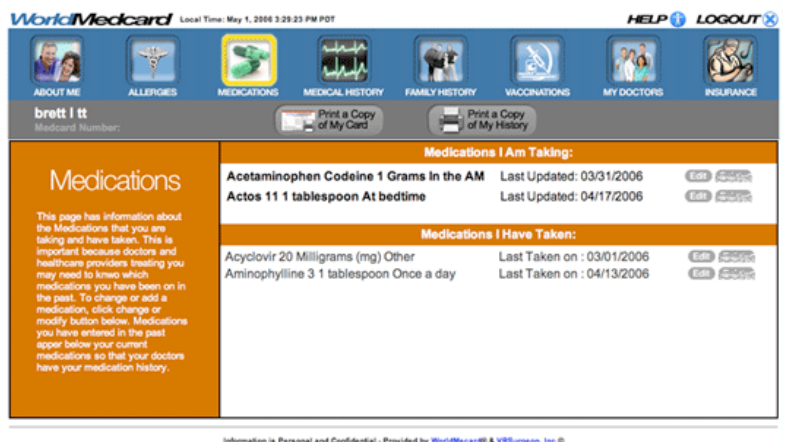

Figure 3: WorldMedCard uses bright colors and large icons.

Participants' comments about aesthetics of the systems varied greatly, even about the same system, indicating that preferences for look and feel can impact general responses to the systems. For example, in describing one of the WorldMedCard sites, one end- 
user participant described it as "grey and depressing" [EU17] while another maligned it for being "too bright." [EU3]. These results indicate that customization should be available for users of PHR just like the myriad of other web services to which they have grown accustomed. It may be easier to start off with neutral colors and a simplistic look, while still offering users with the ability to change color schemes or layouts.

At the same time, any personalization and customization should occur within standard consistent layouts and web elements. Those sites - and areas within sites - that did not conform to user expectations were described as very problematic by both groups. For example, after describing how pressing enter did not log in the user, an end-user noted:

"[The login button] isn't web standard. It doesn't look like a web application or a desktop application!” [EU13].

Along these lines, accessibility features should also be included allowing users with disabilities to customize the systems to be usable for their particular needs. Although web standards for accessibility already exist, the particular concerns of accessing health information should be taken into account when developing accessible PHR.

\subsection{Familiarity and Comfort}

As major software companies branch into Health IT, brand loyalty and familiarity with those companies is likely to impact how users approach these new technologies. End-users reported being comfortable with an interface or knowing intuitively how it works based on their familiarity with other products from the same company (e.g., search, email). Furthermore, many people commented that they could trust a major technology company with their records. WorldMedCard was much less well known as a corporation than Google and Microsoft, often leaving users concerned about the validity and trustworthiness of this unknown service.

The reaction to a well-known brand was not always positive, however. For example, some end-users feared that their health records on Google Health would become publicly searchable because Google is known by many to be a search engine. Similarly, one end-user noted that it was difficult to use HealthVault because, "I forgot about this one because looks too much like a program on Windows" [EU8], wanting a more weblike feel, so that HealthVault can be more distinguishable from other Windows applications. Many end-users expressed concerns over advertising, noting that their familiarity with some corporations led them to believe the PHR would be heavily adbased, even when no advertising was currently present. For example, "I wouldn't want them to push some drug on me in an ad [in the future]" [EU12]. End-users responded negatively to commercialization present in the form of logos from partners as well, with one end-user saying, "Right before I log in, I see ads...I don't like it!'” [EU12].

Despite familiarity with some of the companies behind PHR systems, the relative novelty of these applications was a particularly significant concern for many end-users. One end-user noted, "I just don't want to be the first person to do it. Maybe after thousands" [EU1]. This attitude correlated to concerns from HCI experts, who often commented on the lack of consistency and standards compared to more established web services. Likewise, a major point of concern for many of the clinicians was the integration and standardization required to get multiple providers sharing data. For example, a family medicine physician noted:

"I noticed each of [the sites used in the study] partners with a particular set of vendors of a site to sort of import or export the records... it sure would be nice if everybody could get on the same page..." [MP1]

Many of the sentiments described by both end-users and clinicians echo those of groups such as the National Institute of Standards and Technology (NIST) and the American Medical Informatics Association (AMIA), who have been working towards standardizing Health IT.

\subsection{Collaboration, Communication, and Integration}

Tracking status of the system and information stored in itincluding technological changes, records status, and messaging interfaces - can be a huge challenge in multi-user, data-intensive healthcare technologies. Representations of system status of some kind was present in all three PHR systems evaluated, but the particular implementations were not consistent across systems. For example, Google Health summarized all system status on a primary status page while Microsoft HealthVault displayed individual status messages on each page. WorldMedCard was the only system that chose to disclose only minimal system status $\mathrm{s}$, which failed to alert the end-users when a change occurred. An added challenge to PHR systems over other web-based services involves tracking the status of records on top of the system status. The primary point of concern for all the interviewees was the ability to track whether the lab results were completed and the status of various medical procedures.

All of the end-users in the evaluation welcomed the idea of sharing data with clinicians and many with other individuals as well. For example, one end-user emphasized how she liked the sharing function by saying, "Sharing's easy, plus you can specify how much they see or edit" [EU3]. Some patients wanted to share their data electronically and others by being able to print a comprehensive record to paper from the PHR system, but they all wanted the ability to share. They were concerned, however, about the level of detail in the medical records. Privacy issues are discussed in more detail in the next section.

Aside from end-users, the idea of sharing data among providers also appealed to many clinicians. Collaboration is a key component that many clinicians believed would be helpful in looking through patient records. For example, one clinician made a remark on the importance of information source and the collaborators involved:

$$
\begin{aligned}
& \text { “... what would be important... is to be able to sort of } \\
& \text { glance by some ... visual coding where you can see } \\
& \text { exactly where information comes from." [MP1] }
\end{aligned}
$$

Consideration of communication and collaboration was not just limited to the idea of transferring data. Rather, the majority of clinicians and the end-users requested that PHR records be integrated with official clinical records. Although one clinician interviewed described PHR as " $a$ virtual suitcase... of a patient's own information that he or she [can] release or choose not to release" [MP2] and therefore would not have a need to be integrated with official medical records, most of the clinicians interviewed were more interested in discussing the concept of full integration of PHR with electronic medical records (EMR) than 
patient-originated sharing of data. With this model, medical records can:

\begin{abstract}
"be written by the people who provided the care and who have the training and the knowledge of how to document medical problems. But then the patient [can point out errors]. So there's accountability on both parts. " [MP4]
\end{abstract}

\subsection{Privacy, Security, and Trust}

Privacy and security in any web-based application is always a concern. These concerns can be more profound in the case of patient health information web-based applications, such as PHR [14], where patients are uploading private health information to a server. Certain policies such as HIPAA are to ensure that patient data is protected. The sensitivity of patient data makes privacy and security much of a concern to patients and clinicians alike.

Individual end-users incorporated feelings of trust and security from known corporations into their assessment of the trustworthiness of the PHR. Heavy users of other services provided by the same company (e.g., Google Mail and Calendar) tended to be concerned that the company "already knows a lot about me, so putting my health information is somewhat of a concern" [EU13]. Furthermore, the end-users trust the systems less if the login information used for the PHR is also used for other services provided by the same company (e.g., users of both Google and Microsoft Passport products are able to log into all other services with the same username and password). At the same time, other users commented that unknown companies were not as trustworthy. For example, "I think I may have a bias against companies I don't know-it is your health record" [EU3].

None of the end-users noted the security indicators present in their web browsers, echoing former work showing people do not notice these indicators for general web sites [6]. They did, however, note the security differences they perceived to be inherent to a webbased application as compared to a downloadable application that runs on a specific computer, typically indicating that the latter is more secure.

\section{"If it was a downloadable program, I'd use it, because I don't feel comfortable putting my information on a website" [EU4].}

Finally, the cost of the service impacted perceptions of security and privacy for many users, who often believed that a company not charging for a service (as none of the three PHRs did) would be unlikely to secure the data properly. For example, one end-user said that, "If it were a paid service, I'd feel safer" [EU12]. In this case, the business models are as important to perceptions of security as the systems themselves. This issue brings up the limitations of the systems themselves to influence the perceptions about security and privacy surrounding them.

Generally, clinicians were less concerned than patients about the security of online health records. When they did describe concerns, they often equated them to those inherent to any medical record. For example, the director of an emergency department commented:

"There's always a question about who's going to be the custodian of the record, but really, if they had come from another hospital ... I would attach my hospital's number to [the paper record] ... and actually submit this as part of our records, so, if it were ever an issue, you know, with a court or something, I acted on the best information I had at that time and here's the information I have." [MP2]
These results indicate that not only should data be secure, but its provenance should be included in the record itself. Currently, PHR and other Health IT often do not store this kind of meta-data, but it should be explored as a valid part of the record in terms of both storage and retrieval.

\subsection{Ensuring Accurate Data}

Along with ensuring the data are gathered from reliable sources, participants were naturally considered with ensuring accurate data, meaning both complete and without error. Clinicians consistently touted responsibility for the health of the patient as the most important factor in their feelings about Health IT and PHR. Likewise, patients described their feelings about Health IT as fundamentally linked to its ability to generate positive health outcomes. This shared purpose provided a basis for many participants to trust each party to act appropriately to keep accurate records. Thus, clinicians generally reported not being concerned about patients or their families deliberately altering their records, noting, for example "most patients just want to be cured." [MP2] Some, in particular nurses and medical students as opposed to more senior physicians, described still wanting to question patients for greater depth and nuance:

"I would still want to ask them myself... when we see a patient, we can look at their chart and...say, 'So is this so?' or you know, 'Tell me about this.' "' [MP4]

The minority of clinicians who described still wanting to collect data from patients directly - rather than through PHR - tended to be concerned about their responsibility for the patient. For example, a family care physician commented,

"I have to ask the patient in depth, because if I take over, then that's the care of the patient... all of the responsibility of the well-being of the patient." [MP5]

Linked to the concern for accurate records is a major concern by clinicians and patients alike to avoid medical errors. A small error in a medical record (e.g., a typo or an extra 0 in a dosage) could result in potentially damaging or even life threatening results for a patient. A variety of Health IT systems now have medical error checking features, in which decision support and expert systems aid clinicians in avoiding medical errors (e.g., automated drug dispensing such as bar coding which quickly identifies drug name, dose, administration time, clinician and patient name [7] or handheld mobile devices that allow for communication in the case of a medical abnormality [1]). Although these systems are somewhat commonplace in hospital settings, PHR systems have largely not yet evolved to take advantage of this type of functionality, a concern for many of the participants in this study (e.g., "Blood pressure should not be 10000000000" [HE4]). Furthermore, both end-users and HCI experts were also concerned about the need for improved help and documentation on the sites and the ability to undo errors - in particular, the deletion of information later found to be needed. Finally, unlike many other web services, the PHR systems we evaluated did not save information automatically, leading users to move to a different page without saving their data.

PHR information provided to clinicians can be seen as a burden, in particular when patients deliver a large quantity of unprocessed data that must be analyzed and verified to ensure that its introduction does not increase the risk for medical errors. One family medicine clinician commented: 
"It's difficult ... to bring in 20 pages... it takes a long time to go through it all and make sure that's what they really mean." [MP1]

Along with the obligation to sort and understand this information, clinicians must determine which parts of the information a patient has given them is actually reliable. For example, a pediatrician noted that,

"...sometimes patients, you give them a list of medications, even when I see them in the office, they have no idea what medications they're on." [MP3]

Both patients and clinicians alike described the accuracy of patient data in the PHR as fundamental to their ability to adhere to recommendations (patients) and to prescribe those interventions in the first place (clinicians).

\section{CONCLUSIONS AND FUTURE WORK}

In this work, we uncovered various socio-cultural, clinical, and usability issues related to PHR adoption and use. Although many of these issues cannot be addressed with improved design processes alone, usability concerns can and should be considered earlier in the design process for Health IT. We conducted HE to determine its efficacy as a means for identifying technical usability bugs. Through this evaluation, we were able to identify many important technical usability problems, such as medical error checking. However, there are other issues that still cannot be determined this way, thus a need for a more comprehensive evaluation. Along with HE, we conducted end-user evaluations and clinician interviews. Many of the socio-cultural issues that emerged from end-user evaluations and clinician interviews were not present throughout the HE session. Both the design of PHR and the design process can be improved, leaving open questions for future work.

Because there is just too much information in a health record for a clinician to glance over quickly, simple analysis and summarization would save clinicians an immense amount of time, making them more likely to use PHR generated data. PHR systems must appropriately secure records and provide appropriate access controls to ensure user privacy. Thus, both traditional security and privacy mechanisms must be used alongside new methods for tracking and displaying data provenance. Such an example would be allowing the user to choose if a password is needed to obtain access to certain information. Additionally, PHR can provide more trust in the records than traditional paper records by utilizing advanced computational features, such as Google's conflicting medication checking.

An open challenge for PHR systems is designing them to suit the needs of a wide variety of potential users. Medical terminology may be difficult for patients to understand but significant and relevant to clinicians. PHR systems will need to provide educational or translational information for patients, caregivers, and clinicians to balance these concerns. Another major open challenge is the provision of help to users. Real-time support can be very expensive, a challenge to the many free or low-cost PHR systems currently available. Furthermore, many users will want or need medical help, not just technological, leaving support providers in a difficult position of continually defining their scope of activity and billing and reimbursement systems needing to be reformed to accommodate these new forms of patient care.
PHR have enormous potential for empowering patients, increasing patient-provider communication, and tracking and monitoring health and wellness over time. Tracking these data over extended periods of time has the potential to provide baseline data, making detection of decline more efficient [11]. Furthermore, regular use of PHR can be beneficial in critical care situations, such as a trip to Emergency care, providing up-to-date information for a patient who may not normally be seen at the hospital that is currently providing treatment. Like any novel interactive system, before successful adoption can occur, however, the substantially challenges to usability and usefulness present in these systems must be addressed. Our results indicate that the lack of adoption by significant numbers of end-users and clinicians is in part due to these challenges in the usability of the systems but also in misunderstandings of the context of use by systems designers. Our results further reinforce the need for integrating traditional and more recently developed methods from the HCI literature into the field of medical informatics, in particular in light of designing online systems for the general user population, such as PHR.

Our empirical evidence and subsequent analysis demonstrate some of the barriers to the adoption and use of PHR. These results indicate a variety of ways in which both the design of current PHRs and the design process can be improved. The systems evaluated in this study have continued to evolve, and new systems have emerged. Further research must be conducted as designers and developers continue to innovate, policies encourage more clinical use, and end users become more motivated to use these systems.

\section{ACKNOWLEDGEMENTS}

We thank the participants in our evaluation studies for their time and feedback. We also thank David Nguyen, Khai Truong, Jed Brubaker, Monica Tentori, and Meg Cramer for providing valuable feedback on previous drafts. This work is approved by the UC Irvine IRB under protocol HS\# 2008-6427.

\section{REFERENCES}

[1] Bates, D., Cohen, M., Leape, L., Overhage, M., Shabot, M., Sheridan, T. Reducing the Frequency of Errors in Medicine Using Information Technology. JAMIA, 299-308. 2001.

[2] Berner, E., Detmer D., Simborg D. Will the Wave Finally Break? A Brief View of the Adoption of Electronic Medical Records in the United States. JAMIA, 3-7. 2005.

[3] Brennan, P., Downs, S., Casper, G., Kenron, D. Project HealthDesign: Stimulating the Next Generation of Personal Health Records. Proc. AMIA'07, 70-74.

[4] Friedman, C., Wyatt, J., Ash, J. Evaluation Methods in Biomedical Informatics. Springer, 2006.

[5] Halamka, J., Mandl, K., Tang, P. Early Experiences with Personal Health Records. JAMIA, 1-7. 2008.

[6] Jensen C., Potts C., Jensen C. Privacy Practices of Internet Users: Self-Reports Versus Observed Behavior. International Journal of Human-Computer Studies, 203-227. 2005.

[7] Kaushal, R., Barker, K., Bates, D. How Can Information Technology Improve Patient Safety and Reduce Medication Errors in Children's Health Care? Proc. Archives of Pediatric and Adolescent Medicine'01, 1002-1007. 
[8] Kim, E., Wang, M., Lau, C., Kim, Y. Application and Evaluation of Personal Health Information Management System. Proc. IEMBS'04, 3159-3162.

[9] Krohn R. The Consumer-Centric Personal Health Record It's Time. Journal of Healthcare Information Management, 20-23. 2007.

[10] Lee, M., Delaney, C., Moorhead, S. Building a Personal Health Record from a Nursing Perspective. IJMI, 308-316. 2007.

[11] Morris, M., Intille, S., Beaudin, J. Embedded Assessment: Overcoming Barriers to Early Detection with Pervasive Computing. Proc. Pervasive'05, 333-346.

[12] Nielsen, J., Mack, R. Usability Inspection Methods. John Wiley \& Sons '94.

[13] Personal Health Records: Lots of Interest, but Few Users. http://www.readwriteweb.com/archives/personal_health_reco rds_lots_of_interest_no_users.php. Retrieved March 2009 .

[14] Peters, K., Niebling, M., Green, T., Slimmer, C., Schumacher, R. Consumers Compare Online Personal Health Record (PHR) Applications. User Centric. 2009.
[15] Rindfleisch, T. Privacy, information technology, and healthcare. Communications of the ACM, 92-100. 1997.

[16] Smolij, K., Dun, K. Patient Health Information Management: Searching for the Right Model. Proc. Perspectives in Health Information Management'06, 1-11.

[17] Tang, P., Ash, J., Bates, D., Overhage, M., Sands, D. Personal Health Records: Definitions, Benefits, and Strategies for Overcoming Barriers to Adoption. JAMIA, 121-126. 2005.

[18] Williams, M., Parker, R., Baker, D., Parikh, N., Pitkin, K., Coates, W., Nurss, J. Inadequate Functional Health Literacy Among Patients at Two Public Hospitals. JAMA, 1677-1682. 1995.

[19] Win, K., Susilo, W., Mu, Y. Personal Health Record Systems and Their Security Protection. Journal of Medical Systems, 309-315. 2006.

[20] Wolter, J., Friedman, B. Health records for the people: touting the benefits of the consumer-based personal health record. Journal of AHIMA, 28-32. 2005. 